\title{
High Harmonic Phase in Molecular Nitrogen
}

\author{
B. K. McFarland, J. P. Farrell, P. H. Bucksbaum, and M. Gühr* \\ PULSE Institute, SLAC National Accelerator Laboratory, Menlo Park, \\ CA 94025, USA, and Departments of Physics and Applied Physics, \\ Stanford University, Stanford, CA 94305, USA.
}

\begin{abstract}
Electronic structure in atoms and molecules modulates the amplitude and phase of high harmonic generation (HHG). We report measurements of the high harmonic spectral amplitude and phase in $\mathrm{N}_{2}$. The phase is measured interferometrically by beating the $\mathrm{N}_{2}$ harmonics with those of an Ar reference oscillator in a gas mixture. A rapid phase shift of $0.2 \pi$ is observed in the vicinity of the HHG spectral minimum, where a shift of $\pi$ had been presumed [J. Itatani et al., Nature 432, 867 (2004)]. We compare the phase measurements to a simulation of the HHG recombination step in $\mathrm{N}_{2}$ that is based on a simple interference model. The results of the simulation suggest that modifications beyond the simple interference model are needed to explain HHG spectra in molecules.
\end{abstract}

Work supported in part by US Department of Energy contract DE-AC02-76SF00515 
High Harmonic Generation (HHG) is used as a light source in the vacuum ultraviolet (vuv) and soft x-ray regions [1-3] as well as for the generation of attosecond pulses [4, 5]. The amplitude of strong field high harmonics from a molecule has been used to determine the electronic structure of a molecular orbital [6]. Electronic structure interpretations of the HHG spectrum are critically dependent on the effects of the high intensity laser field on the orbital structure and the HHG process.

In the established model of HHG [7-9] a laser-induced potential deforms the atomic or molecular potential resulting in the tunnel ionization of a free-electron wave packet $\psi_{\text {free }}$

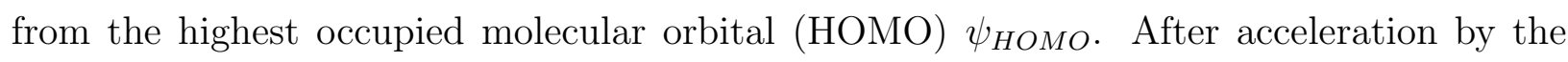
electric field of the laser the electron wave packet can recombine to the atom or molecule and releases a photon with an energy given by the kinetic energy gained in the field plus the ionization potential. The recombination process is described by the dipole matrix element $\left\langle\psi_{\text {free }}|e z| \psi_{\text {HOMO }}\right\rangle$.

A quantum mechanical calculation of this dipole matrix element for $\mathrm{H}_{2}$ and $\mathrm{H}_{2}^{+}$using the time-dependent Schrodinger equation predicts the HHG amplitude and phase for this system $[10,11]$. Both the amplitude and phase are modulated by interference in the recombination probability. The interference depends upon the transition dipole matrix element between the continuum state of the returning electron and the bound state of the recombined electron. The modulation pattern in these calculations resembles a simple two-center wave interference, where destructive interference leads to a minimum accompanied by a phase shift of $\pi$ radians.

Amplitude minima due to destructive interference have been claimed in measurements of a number of simple diatomic and triatomic systems [12-15]; however there have been few accompanying studies of the harmonic phase, which is expected to display a rapid shift in the vicinity of a minimum [15]. A modification of the two center model for $\mathrm{N}_{2}$ based on the actual electron density of the molecular HOMO has been presented [16]. The model predicted a minimum in the harmonic spectrum around the 25 th harmonic of $\mathrm{N}_{2}$ in the case that the internuclear axis is parallel to the polarization of the laser pulse driving the HHG. The phase of harmonics from unaligned $\mathrm{N}_{2}$ was measured, but the measurement did not cover the whole range of harmonics needed to resolve the $\mathrm{N}_{2}$ phase jump [17]. Phases are particularly important for the reconstruction of the molecular orbital in the HHG orbital tomography method. In orbital tomography [6], the recombining electron wave function is 
approximated as a plane wave, thereby neglecting the ionic potential of the ionized molecule. The molecular dipole formed upon recombination of the plane wave imprints its phase and amplitude on the emitted harmonic light. This dipole is related to the wave-vector $\mathrm{k}$ of the recombining electron by the free electron dispersion relation. The dipole in k-space and the molecular orbital in position space are related by a Fourier transformation. For orbital tomography, one measures the dipole in $2 \mathrm{~d} \mathrm{k}$-space by rotating the molecules with respect to the recombining electron. To reconstruct the molecular HOMO in two dimensions an inverse $2 \mathrm{~d}$ Fourier transformation is performed, that is not well-defined without phase.

A common criticism of orbital tomography is the use of plane waves. For Ar it is known that a plane wave treatment results in a correct harmonic phase jump magnitude, however at the wrong spectral position [18]. The inclusion of the Coulomb potential for $\mathrm{H}_{2}^{+}$has resulted in a reduced harmonic phase jump in comparison to the predicted $\pi$ phase jump of a plane wave model [19]. The first realization of tomography in $\mathrm{N}_{2}$ assumed a phase change of $\pi$ and modelled the recombining electron as a plane wave in the orbital reconstruction [6]. This orbital reconstruction also only considered harmonic radiation from the HOMO. Recent experiments show, that also the HOMO-1 contributes to $\mathrm{HHG}$ in $\mathrm{N}_{2}$ [20] and its influence on the orbital tomography method needs to be clarified.

The harmonic phase can be measured with the RABBITT techinique which relies on measurements of electron emission in the presence of the high harmonic and the generating infrared laser fields $[15,17,21]$. Alternatively, one can determine the relative phase between two species in an interference experiment [22, 23]. As in [23], we use a rare gas to obtain information about the phase of molecular HHG. To match the intrinsic phase due to the electron propagation, the ionization potentials IP of the molecule and the rare gas are matched. The IP of Ar $(15.8 \mathrm{eV})$ closely matches that of $\mathrm{N}_{2}(15.6 \mathrm{eV})$.

The Ar high harmonic spectrum has a pronounced phase feature in the energy range accessible in our experiments, which is due to a Cooper minimum [24]. The Cooper minimum in Ar is usually observed in photoionization spectra [25]. It is also visible in high harmonic spectra [6, 26-29], but it has rarely recieved comment in the past [30]. Recent measurements have led to further discussion of the Cooper minimum in the HHG spectrum [31, 32]. We show that the Cooper minimum in the HHG spectrum is accompanied by a $\pi$ phase jump. This was predicted by the general theory [18, 24, 33], but has never been measured in photoionization or HHG studies. This illustrates how vuv photoionization is the inverse 


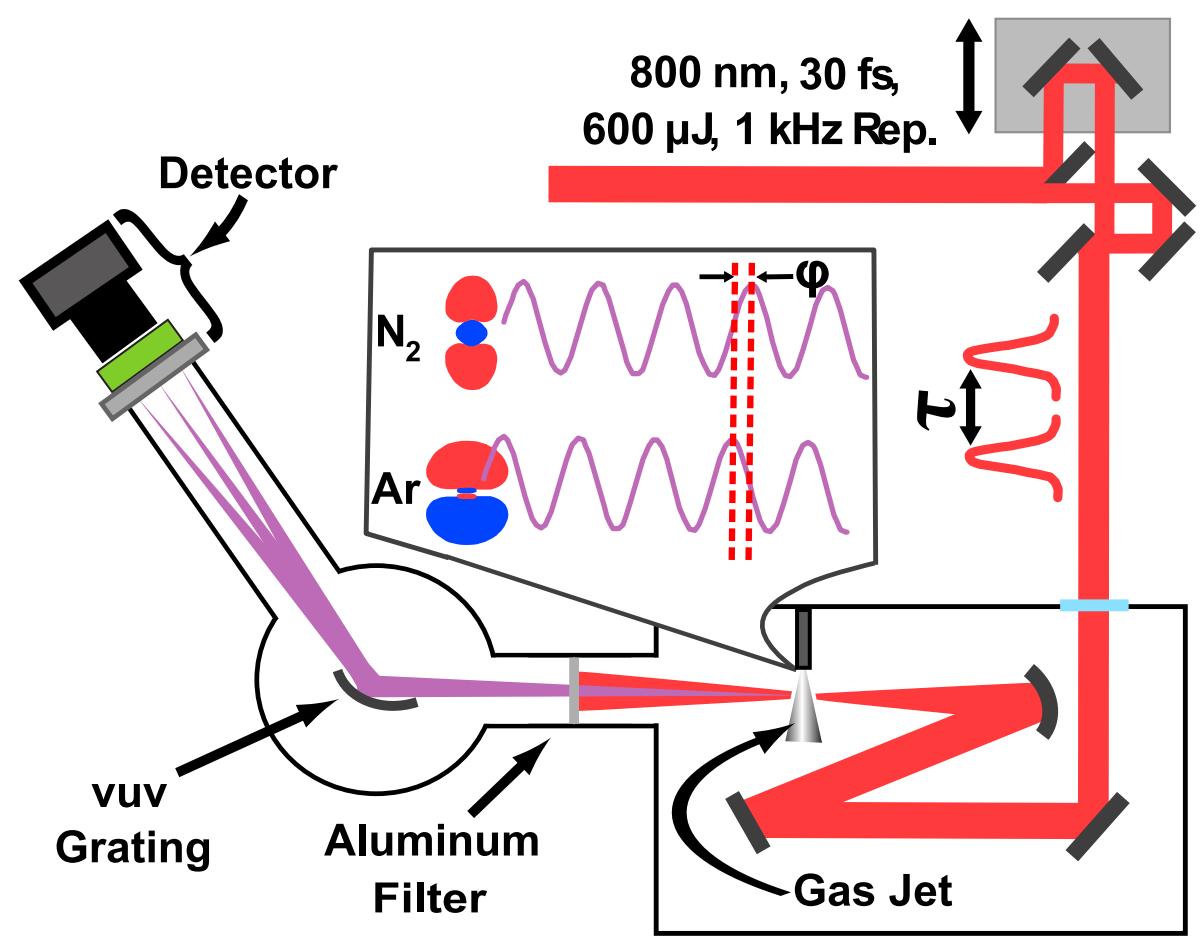

FIG. 1: (Color online) High Harmonic Generation setup. The laser beam is split into time delayed pump-and probe pulses in a pulse front beam splitter. The two beams are recombined on a molecular jet from a supersonic expansion in vacuum. One pulse aligns the sample, and the other one creates high harmonics on the aligned sample. The high harmonics are dispersed in a vuv spectrometer and detected on an MCP-phosphor screen assembly.

process of the recombination step in HHG.

We measure the relative phase between $\mathrm{Ar}$ and $\mathrm{N}_{2}$ and use this information to learn about the Ar and $\mathrm{N}_{2}$ phase features. A significant finding is that there is a rapid phase change in the vicinity of the spectral minimum for $\mathrm{N}_{2}$, but this change is not $\pi$ radians as predicted by a "two-center interference" model. We perform simulations taking the aligned ensemble and full electron orbital into account, and compare to our experimental results.

A diagram of our experimental setup is given in Fig. 1. A chirped-pulse amplifier laser system provides a $1 \mathrm{kHz}$ train of $30 \mathrm{fs}$ pulses centered at $800 \mathrm{~nm}$ with approximately $600 \mu \mathrm{J}$ per pulse. The laser beam is separated into two parts using a split mirror. Both beams are then focused by a spherical mirror $(\mathrm{R}=0.8 \mathrm{~m})$ into a supersonically cooled gas jet inside the HHG chamber. One beam passes through a delay arm and aligns the $\mathrm{N}_{2}$ via impulsive excitation of a rotational molecular wave packet (aligning laser intensity in the 
$10^{13} \mathrm{~W} / \mathrm{cm}^{2}$ range) [34]. The other beam (intensity $\mathrm{I}_{G} \approx 2.3 \times 10^{14} \mathrm{~W} / \mathrm{cm}^{2}$ ) generates HHG on the aligned ensemble. The rotational wave packet evolves following excitation resulting in maximal field-free alignment after at the quantum half-revival (4.2 ps). The alignment results in an enhancement of the HHG signal throughout the entire spectrum. The alignment pulse does not have any influence on the HHG spectra of Ar, and was blocked during the Ar measurements.

We perform a quantum mechanical simulation of the $\mathrm{N}_{2}$ rotational wavepacket as a function of time after the alignment pulse. From the $\left\langle\cos ^{4} \theta\right\rangle$ of these alignment distributions, we reconstruct the time dependent harmonic signal [35]. We optimize the $\mathrm{N}_{2}$ rotational temperature in the simulation to match the temporal characteristics of the experimental HHG signal and find a best fit at $60 \mathrm{~K}$. For these conditions the simulations deliver a $\left\langle\cos ^{4} \theta\right\rangle$ value of $0.25\left(\left\langle\cos ^{2} \theta\right\rangle\right.$ of 0.39$)$ at the alignment maximum.

Optimal phase matching enhances the conversion of the $800 \mathrm{~nm}$ radiation to high harmonics when the jet position is about $2 \mathrm{~mm}$ beyond the focus [36, 37]. The harmonics and $800 \mathrm{~nm}$ radiation are incident on a $100 \mathrm{~nm} \mathrm{Al} \mathrm{filter.} \mathrm{This} \mathrm{blocks} \mathrm{all} \mathrm{radiation} \mathrm{below} 20 \mathrm{eV}$ photon energy from entering the spectrometer chamber immediately beyond the filter. An aperture is used to pass only the portion of the harmonics which travel close to the beam propagation axis. These harmonics are thought to be produced by the short electron recombination trajectories in the atom or molecule, which are more closely phase matched in the forward direction. The long trajectories tend to diverge from the axis of the beam because of phase matching considerations $[38,39]$. The spectrometer disperses the light by means of a variable line-spaced flat-field toroidal grating . The dispersed image is then captured by a vuv detector and image intensifier consisting of a bare microchannel plate followed by a phosphor screen, which is viewed by a CCD camera. The spectrum is calibrated by tightly focusing the femtosecond laser pulses into $\mathrm{Ar}, \mathrm{Kr}, \mathrm{Ne}$, and $\mathrm{N}_{2}$ gas targets and recording the plasma emission with the spectrometer [40].

In order to obtain comparable amplitude data from different gases, we set the MCP voltage to a fixed value and vary the camera acquisition time to adapt to the HHG signal level. For the relative phase measurements of $\mathrm{Ar}$ and $\mathrm{N}_{2}$ we first measure a signal that is proportional to the time integrated intensities of the pure gases $\left(I_{A r}\right.$ and $\left.I_{N_{2}}\right)$ for each harmonic at a fixed gas density. The dependence of HHG on gas density is used to make sure that we are operating in the sub-critical phase matching regime for HHG. We confirm 
the expected quadratic dependence of the HHG signal with gas density over an order of magnitude in backing pressure. To assure detector linearity for large signal levels we use a Peltier cooled vuv CCD camera. Any separation of the gas mixture due to the mass difference between the two species is negligible and thus has no effect on the ratio of Ar and $\mathrm{N}_{2}$ [41]. The harmonic intensity generated in a gas mixture at fixed number density with a fraction $\mathrm{r}_{A r}$ of $\mathrm{Ar}$ and $\mathrm{r}_{N_{2}}$ of $\mathrm{N}_{2}$ is given by:

$$
I_{m i x}(\rho)=N^{2}\left|r_{A r} \sqrt{I_{A r}(\rho)}+r_{N_{2}} \sqrt{I_{N_{2}}(\rho)} e^{i \phi}\right|^{2}
$$

where $N$ is the number of overall atoms and $\mathrm{I}_{m i x}$ is the $\mathrm{HHG}$ intensity from the mixture. By determining the relative signal strengths of the mixture, Ar, and $\mathrm{N}_{2}$ from our measurement at the same density $\rho$, we can deduce the relative phase $\phi$ between $\mathrm{Ar}$ and $\mathrm{N}_{2}$ for every harmonic.

HHG spectra of $\mathrm{N}_{2}$ at the revival and Ar are shown in Fig. 2a corrected for the spectrometer efficiency (by grating manufacturer [42]) and that of the MCP detector [43]. The intensity of the high harmonic generating pulse $\mathrm{I}_{G}$ was calibrated from the spectral cutoff. The $\mathrm{N}_{2}$ spectrum exhibits a shallow minimum at harmonic 25. In Ar this minimum is absent, but there is a pronounced minimum around harmonic 33. We did not find any phase matching dependence of this minimum by scanning the position of the Ar gas jet in the laser focus. We attribute the Ar spectral dip to a Cooper minimum [24]. A $\pi$ phase jump of the recombination matrix element is expected at the amplitude minimum [18, 24].

With that in mind, we turn to the measurements of the relative phase between harmonics generated in $\mathrm{Ar}$ and aligned $\mathrm{N}_{2}$. The measured harmonic spectra for Ar (dashed,dark grey line), aligned $\mathrm{N}_{2}$ (solid,light grey line), and a 1:1 mixture of Ar and aligned $\mathrm{N}_{2}$ (dotted, black line) in Fig. 2a were evaluated according to Eq. 1, with $r_{A r}=r_{N_{2}}=0.5$. An expression for the cosine of the relative phase $\phi$ is found by evaluating the right hand side of Eq. 1. The values for $\cos \phi$ measured in the experiment are given in Fig. 2b. The values of $\cos \phi$ greater than one can be explained by the increased vuv transmission of Ar around the Cooper minimum. In the gas mixture the transmission window of Ar around the spectral amplitude minimum will result in greater transmission of the $\mathrm{N}_{2}$ harmonics. We modify Eq. 1 to include transmission functions for $\mathrm{Ar}$ and $\mathrm{N}_{2}$, and find that values of $\cos \phi$ greater than one are possible. However, the exact magnitude of this effect is difficult to model since we do not 


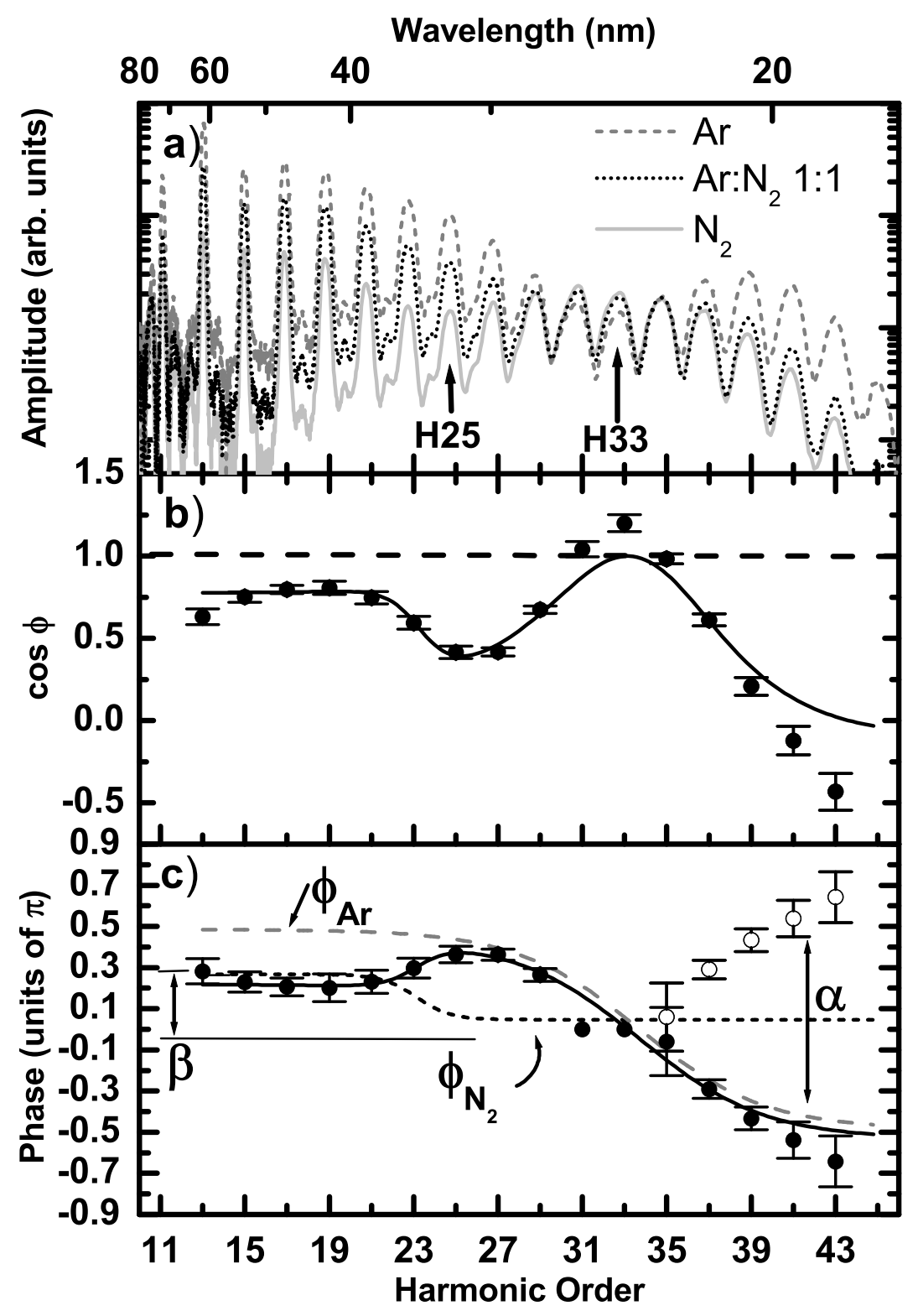

FIG. 2: a) HHG spectra for Ar, aligned $\mathrm{N}_{2}$ and a 1:1 mixture of $\mathrm{Ar}$ and aligned $\mathrm{N}_{2}$. b) $\cos \phi$ values of the relative phase between the $\mathrm{Ar}$ and $\mathrm{N}_{2}$ harmonics deduced via Eq. 1 with a fitted black solid line. Values of $\cos \phi$ larger than 1 indicate a systematic effect in the measurement, probably related to the sharp Ar transmission increase around the Cooper minimum. c) Values for the relative phase $\phi$ with a solid black line fitted and the anticipated phases $\phi_{A r}$ for Ar (dashed grey line) and $\phi_{N_{2}}$ for aligned $\mathrm{N}_{2}$ (dotted black line). The amplitudes of the phase jumps are given by the parameters $\beta$ and $\gamma$ for $\mathrm{N}_{2}$ and Ar, respectively. 
have precise knowledge of the gas density profile present in the experiment. We determine the relative phase $\phi$ between the $\mathrm{Ar}$ and $\mathrm{N}_{2}$ harmonics from the $\cos \phi$ data in Fig. $2 \mathrm{~b}$ and plot them in Fig. 2c. Due to the sign ambiguity of the inverse cosine, the relative phase is plotted both positive and negative after it approaches zero at harmonic 33 . The phase ambiguity will be discussed further below.

In the phase decomposition, we assume that the attochirp [21] is the same for Ar and aligned $\mathrm{N}_{2}$, due to similar ionization potentials. In the spirit of the strong field approximation (SFA), we thereby also neglect the exact form of the ionic potential in the propagation of the electron. This allows us to attribute features in the relative phase measurement to the phase jumps in $\mathrm{Ar}$ and $\mathrm{N}_{2}$.

Neither the $\mathrm{N}_{2}$ nor the Ar HHG phase has been determined in our spectral range. However, the $\mathrm{N}_{2}$ and Ar amplitude modulations appear in different regions of the HHG spectrum, as seen in the HHG amplitude spectra in Fig. 2a. The relative phase is dominated by two spectrally separate phase jumps centered near harmonics 25 and 33 (see Fig. 2c). We attribute the phase shift at harmonic 25 to a phase jump in the $\mathrm{N}_{2}$ harmonics since it is centered at the same position as the amplitude minimum in the $\mathrm{N}_{2}$ spectrum. We attribute the phase jump at harmonic 33 to the Cooper minimum in the Ar HHG amplitude spectrum. In order to disentangle the $\mathrm{N}_{2}$ and Ar phases from the relative phase we assume the phase jumps to be localized around the respective amplitude minima and flat elsewhere. This is reasonable judging from the widths of the spectral amplitude minima and their spectral separation. Guided by the $\pi$ phase jump of the Cooper minimum we assume $\phi_{A r}$ to jump to negative phases above the 33th harmonic thereby rejecting the phases given by open circles in Fig. 2c.

We use a nonlinear least squares fitting function to extract the $\mathrm{N}_{2}$ and $\mathrm{Ar}$ phases from our measurement. The $\mathrm{N}_{2}$ and Ar phase jumps are modeled by hyperbolic tangents. The difference between the two hyperbolic tangent functions is fit to our measurement. The position, width, and magnitude of the $\mathrm{N}_{2}$ and Ar phase jumps are used as fitting parameters. We define the width of the phase jump by the energies where the phase reaches 0.7 of the asymptotic value on each side of the phase jump. Based on the observation of spectrally separate phase jumps, we constrain the width of the Ar phase jump to the width of the Ar amplitude minimum (estimated to be $16 \mathrm{eV}$ ). The fit is given as the black solid line in Fig. 2c and provides a consistent explanation of the relative phase $\phi$ based on the phase 
jumps of $\mathrm{Ar}$ and $\mathrm{N}_{2}$. The individual $\mathrm{N}_{2}$ and $\mathrm{Ar}$ contributions to the phase are given by the black dotted and the gray dashed lines, respectively. The fit gives an $\mathrm{N}_{2}$ phase jump of $0.2 \pi$ centered near harmonic 23 which spans about $4 \mathrm{eV}$. The phase jump of Ar has an amplitude of $\pi$ centered at harmonic 33. The width of the fitted $\mathrm{N}_{2}$ phase jump closely matches the width of the $\mathrm{N}_{2}$ amplitude minimum. Also, the spectral positions of the $\mathrm{N}_{2}$ and Ar phase jumps closely match the positions of the amplitude minima. The fit to the relative phase for the lowest harmonics (harmonics 13 and 15) and the highest harmonics (harmonics 39 through 43) deviates from the experimental data. It is possible that unmatched attochirps lead to such a behavior.

Fully quantum mechanical calculations of $\mathrm{HHG}$ from $\mathrm{H}_{2}$ and $\mathrm{H}_{2}^{+}$molecules predict phase jumps of slightly less than $\pi$ [10]. A calculation of the phase in unaligned $\mathrm{N}_{2}$ has been reported in [17], however, to our knowledge no calculation of the high harmonic phase in aligned $\mathrm{N}_{2}$ has been performed that can be compared to our results.

We compare our experimental phase results for $\mathrm{N}_{2}$ to a semi-classical simulation performed within the framework of the SFA, which neglects the potential of the molecular ion during the electron propagation. We concentrate on electron recombination events that result in phase matched radiation with small divergence angles, the so called short trajectories $[36,37]$. We integrate the electron phase along each trajectory and construct a plane wave representation of the recombining electron for a laser pulse intensity of $\mathrm{I}_{G}=2.7 \times 10^{14} \mathrm{~W} / \mathrm{cm}^{2}$. This electron wave is superimposed with the $\mathrm{N}_{2} \mathrm{HOMO}$ resulting in a time dependent dipole described by the dipole matrix element $\left\langle\Psi_{\text {HOMO }}|e z| \Psi_{\text {free }}(t)\right\rangle$ [16]. The dipole matrix element is only calculated in the window of one single laser cycle. The HOMO orbital used in the simulation is calculated with the STO-3G basis in the Gaussian software package [44].

The alignment of the internuclear axis with respect to the recombining electron wave $\mathrm{k}$ vector influences the recombining dipole. The ionization and recombination dependence on the alignment distribution have opposite effects on the spectral position of the phase jump. Recombination causes the HHG minimum to shift to higher harmonics for large angles $\alpha$ between the internuclear axis and the recombining electron k-vector because the HOMO lobes appear closer together in the direction of the recombining electron. A broad alignment distribution contains many molecules standing at large angles $\alpha$ with respect to the recombining electron k-vector, moving the HHG minimum and phase jump to higher harmonic energies in the recombination step. However, molecules at large $\alpha$ ionize less resulting in a 
smaller contribution to HHG from these molecules, pushing the HHG minimum and phase jump to lower harmonic energies $[45,46]$. Therefore ionization effects are included explicitly in the calculation. We integrate the dipole matrix elements for different $\alpha$ according to the calculated angular distribution at the half revival [47]. The ionization is modeled by weighting the dipole matrix elements with the corresponding MO-ADK ionization probability [45]. The weighted dipole matrix elements are then coherently superimposed [14, 48].

We Fourier transform the superimposed dipole matrix elements and multiply the absolute value by $\omega^{4}$ to find the HHG spectral intensity [49]. We observe a minimum and an associated phase jump in the $\mathrm{N}_{2}$ HHG spectrum. The shape and position of the minimum and phase jump in the Fourier domain are very sensitive to the shape of the temporal window in which the dipole was calculated $[50,51]$. Therefore, we perform the phase calculation in the time domain and find the phase of the HHG radiation by calculating the phase of the time dependent dipole acceleration. We attribute recombination times to energies using the classical trajectory approach [7]. To improve the visibility of the phase jump, we subtract the attochirp of the recombining electron wave from the time dependent dipole phase. This attochirp is calculated by performing the same HHG simulation for a Gaussian shaped ground state wavefunction which has the same IP.

The simulated relative phase between the HHG from the $\mathrm{N}_{2} \mathrm{HOMO}$ and the reference atom is given in Fig. 3. A hyperbolic tangent fit to the simulated curve gives a jump of approximately $0.7 \pi$ that covers a spectral range spanning $10 \mathrm{eV}$ (6 harmonics) centered near $45 \mathrm{eV}$ (harmonic 29). In comparison, the experimental relative phase measurement gives a phase jump of $0.2 \pi$ and a width of 3 harmonics $(4 \mathrm{eV}$ ) centered near harmonic 23 .

Concerning the position of the phase jump, it is well known that the SFA delivers a spectral position that differs from the experimental value [18]. The SFA is also predicted to influence the amplitude of the phase jump. A recent simulation of the $\mathrm{HHG}$ in $\mathrm{H}_{2}^{+}$ approximates the effect of the molecular potential by representing the ionized electron as a two-center coulomb continuum wavefunction [19]. The results of the simulation predict a phase jump of $0.6 \pi$ compared to the $\pi$ phase jump predicted when modeling the electron as a plane wave. This might help explain why the simulated $\mathrm{N}_{2}$ phase jump is large compared to the measured phase jump.

In addition, HHG contributions from more deeply bound orbitals can influence the magnitude and position of the phase jump in $\mathrm{N}_{2}$. It was recently suggested that features in 


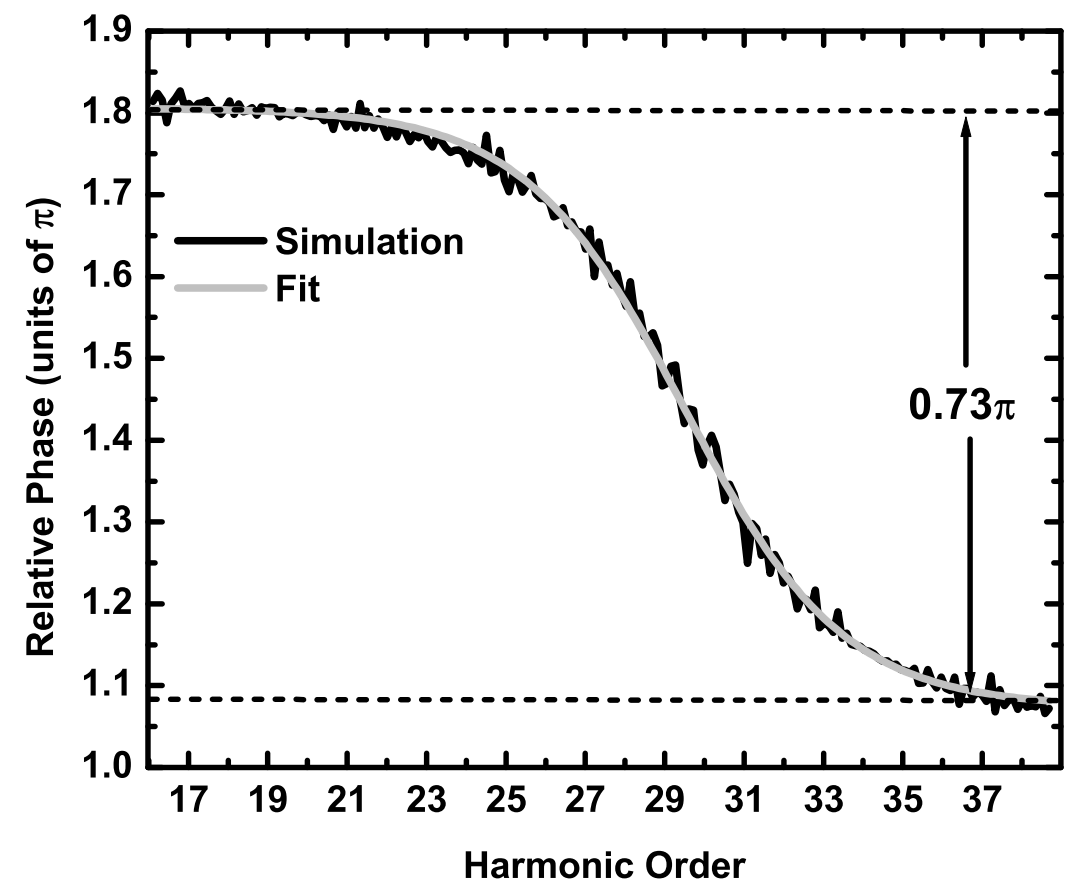

FIG. 3: Simulated relative phase between $\mathrm{N}_{2}$ and a reference atom giving a phase jump of $0.73 \pi$ centered at near harmonic 29.

the HHG spectrum due to the electronic structure of the HOMO may be obscured due to multiorbital contributions [52]. It has also been observed in $\mathrm{N}_{2}$ that orbitals lying energetically below the HOMO, in particular the HOMO-1, also contribute to HHG in molecules [20]. Recent theoretical and experimental studies of the HHG polarization provide additional support for multiorbital effects [52-54]. The experimental phase determined for $\mathrm{N}_{2}$ may have both HOMO and HOMO-1 contributions. Near the amplitude minimum in the HOMO dipole of $\mathrm{N}_{2}$, even a small signal of the HOMO- 1 orbital could dominate the spectrum. There is also the possibility that orbitals energetically lower than the HOMO-1 may contribute to the overall HHG signal [55].

To further explore the effects of alignment on the phase jump we simulate the phase for an isotropic alignment distribution $\left(\left\langle\cos ^{2} \theta\right\rangle\right.$ of $\left.1 / 3\right)$, the distribution reached in the experiment with $\left\langle\cos ^{2} \theta\right\rangle$ of 0.39 , and the perfect alignment with $\left\langle\cos ^{2} \theta\right\rangle$ of 1 . The magnitude of the phase jump is very similar in the three cases. What changes notably is the harmonic energy around which the phase jump is centered. The HHG phase jump from the isotropic 
distribution is shifted to higher energies by 3 harmonics $(4.5 \mathrm{eV})$, while the phase jump from perfectly aligned molecules is shifted to lower energies by approximately 10 harmonics (15 eV) compared to the phase jump with the experimental alignment distribution.

In summary, we have measured the spectral phase shift in the $\mathrm{N}_{2}$ HHG spectrum associated with the spectral minimum near harmonic 25 of the $800 \mathrm{~nm}$ fundamental field. We have also measured a phase shift in atomic Ar associated with the Cooper minimum at harmonic 33. We measure a phase jump in $\mathrm{N}_{2}$ of $0.2 \pi$ and a phase jump of $\pi$ in Ar. The spectral position of each phase jump coincides with the position of a minimum in the HHG amplitude spectrum. While the phase jump in Ar is consistent with theoretical predictions, the size of the phase jump measured in $\mathrm{N}_{2}$ is smaller than predicted by a simulation conducted within the confines of the strong field approximation. This suggests that other mechanisms such as the molecular potential and multielectron effects may contribute to the HHG spectral phase near the amplitude minimum in $\mathrm{N}_{2}$, and require a more complete theoretical model of HHG. The magnitude and width of the phase jumps in the harmonic spectrum contain critical information about electronic structure in these quantum systems. We plan to investigate these further.

We thank S.I. Chu, H. Kapteyn, A.T. Le, C.D. Lin, H. Merdji, M. Murnane, O. Smirnova, V. Usachenko, and X. Zhou for very interesting discussions. This paper was written with support from the U.S. Department of Energy, Office of Basic Energy Sciences.

* E-mail:mguehr@stanford.edu

[1] A. L'Huillier and P. Balcou, Phys. Rev. Lett. 70, 774 (1993).

[2] R. L. Sandberg, C. Song, P. W. Wachulak, D. A. Raymondson, A. Paul, B. Amirbekian, E. Lee, A. E. Sakdinawat, C. La-O-Vorakiat, M. C. Marconi, et al., Proc. Natl. Acad. Sci. USA 105, 24 (2008).

[3] A. Ravasio, D. Gauthier, F. R. N. C. Maia, M. Billon, J. P. Caumes, D. Garzella, M. Géléoc, O. Gobert, J. F. Hergott, A. M. Pena, et al., Phys. Rev. Lett. 103 (2009).

[4] P. B. Corkum and F. Krausz, Nature Phys. 3, 381 (2007).

[5] F. Krausz and M. Ivanov, Rev. of Mod. Phys. 81, 163 (2009).

[6] J. Itatani, J. Levesque, D. Zeidler, H. Niikura, H. Pepin, J. C. Kieffer, P. B. Corkum, and 
D. M. Villeneuve, Nature 432, 867 (2004).

[7] P. B. Corkum, Phys. Rev. Lett. 71, 1994 (1993).

[8] K. C. Kulander, K. J. Schafer, and J. L. Krause, Laser Physics 3, 359 (1993).

[9] K. J. Schafer, B. Yang, L. F. DiMauro, and K. C. Kulander, Phys. Rev. Lett. 70, 1599 (1993).

[10] M. Lein, N. Hay, R. Velotta, J. P. Marangos, and P. L. Knight, Phys. Rev. Lett. 88, 183903 (2002).

[11] M. Lein, N. Hay, R. Velotta, J. P. Marangos, and P. L. Knight, Phys. Rev. A 66, 023805 (2002).

[12] C. Vozzi, F. Calegari, E. Benedetti, J. P. Caumes, G. Sansone, S. Stagira, M. Nisoli, R. Torres, E. Heesel, N. Kajumba, et al., Phys. Rev. Lett. 95, 153902 (2005).

[13] T. Kanai, S. Minemoto, and H. Sakai, Nature 435, 470 (2005).

[14] X. Zhou, R. Lock, W. Li, N. Wagner, M. M. Murnane, and H. C. Kapteyn, Phys. Rev. Lett. 100 (2008).

[15] W. Boutu, S. Haessler, H. Merdji, P. Breger, G. Waters, M. Stankiewicz, L. J. Frasinski, R. Taieb, J. Caillat, A. Maquet, et al., Nature Phys. 4, 545 (2008).

[16] M. Gühr, B. K. McFarland, J. Farrell, and P. H. Bucksbaum, J. Phys. B 40 (2007).

[17] H. Wabnitz, Y. Mairesse, L. Frasinski, M. Stankiewicz, W. Boutu, P. Breger, P. Johnsson, H. Merdji, P. Monchicourt, P. Salières, et al., E. Phys. J. D 40, 305 (2006).

[18] A. T. Le, T. Morishita, and C. D. Lin, Phys. Rev. A 78, 023814 (2008).

[19] M. F. Ciappina, C. C. Chirila, and M. Lein, Phys. Rev. A 75, 043405 (2007).

[20] B. K. McFarland, J. P. Farrell, P. H. Bucksbaum, and M. Gühr, Science 322, 1232 (2008).

[21] Y. Mairesse, A. de Bohan, L. J. Frasinski, H. Merdji, L. C. Dinu, P. Monchicourt, P. Breger, M. Kovacev, R. Taieb, B. Carre, et al., Science 302, 1540 (2003).

[22] T. Kanai, E. J. Takahashi, Y. Nabekawa, and K. Midorikawa, Phys. Rev. Lett. 98, 153904 (2007).

[23] N. Wagner, X. Zhou, R. Lock, W. Li, A. Wuest, M. Murnane, and H. Kapteyn, Phys. Rev. A 76, 061403 (2007).

[24] J. W. Cooper, Phys. Rev. 128, 681 (1962).

[25] J. Samson and W. Stolte, J. El. Spect. Rel. Phen. 123, 265 (2002).

[26] J. Zhou, J. Peatross, M. M. Murnane, and H. C. Kapteyn, Phys. Rev. Lett. 76, 752 (1996).

[27] H. J. Shin, D. G. Lee, Y. H. Cha, K. H. Hong, and C. H. Nam, Phys. Rev. Lett. 83, 2544 
(1999).

[28] C. Altucci, R. Velotta, J. P. Marangos, E. Heesel, E. Springate, M. Pascolini, L. Poletto, P. Villoresi, C. Vozzi, G. Sansone, et al., Phys. Rev. A 71, 1 (2005).

[29] P. Colosimo, G. Doumy, C. I. Blaga, J. Wheeler, C. Hauri, F. Catoire, J. Tate, R. Chirla, A. M. March, G. G. Paulus, et al., Nature Phys. 4, 386 (2008).

[30] C. G. Wahlström, J. Larsson, A. Persson, T. Starczewski, S. Svanberg, P. Salières, P. Balcou, and A. L'Huillier, Phys. Rev. A 48, 4709 (1993).

[31] S. Minemoto, T. Umegaki, Y. Oguchi, T. Morishita, A. T. Le, S. Watanabe, and H. Sakai, Phys. Rev. A 78, 061402 (2008).

[32] H. Worner, H. Niikura, J. Bertrand, P. Corkum, and D. Villeneuve, Phys. Rev. Lett. 102, 103901 (2009).

[33] J. Mauritsson, M. Gaarde, and K. Schafer, Phys. Rev. A 72, 013401 (2005).

[34] H. Stapelfeldt and T. Seideman, Rev. of Mod. Phys. 75, 543 (2003).

[35] S. Ramakrishna and T. Seideman, Phys. Rev. Lett. 99, 113901 (2007).

[36] P. Salières, A. L'Huillier, and M. Lewenstein, Phys. Rev. Lett. 74, 3776 (1995).

[37] P. Balcou, P. Salières, A. L'Huillier, and M. Lewenstein, Phys. Rev. A 55, 3204 (1997).

[38] M. Bellini, C. Lynga, A. Tozzi, M. B. Gaarde, T. W. Hansch, A. L'Huillier, and C. G. Wahlström, Phys. Rev. Lett. 81, 297 (1998).

[39] H. Merdji, M. Kovacev, W. Boutu, P. Salières, F. Vernay, and B. Carre, Phys. Rev. A 74, 43804 (2006).

[40] J. P. Farrell, B. K. McFarland, P. H. Bucksbaum, and M. Gühr, Opt. Express 17, 15134 (2009).

[41] G. Scoles, Atomic and Molecular Beam Methods: Volume1 (Oxford University Press, New York, NY, USA, 1988).

[42] S. Kane, private communication, Horiba Jobin-Yvon.

[43] R. Hemphill, J. Edelstein, and D. Rogers, Applied Optics 36, 1421 (1997).

[44] M. J. Frisch et al., Gaussian 03, Revision C.02, Gaussian, Inc., Wallingford, CT, 2004.

[45] X. M. Tong, Z. X. Zhao, and C. D. Lin, Phys. Rev. A 66, 33402 (2002).

[46] D. Pavičić, K. F. Lee, D. M. Rayner, P. B. Corkum, and D. M. Villeneuve, Phys. Rev. Lett. 98, 243001 (2007).

[47] J. Ortigoso, M. Rodriguez, M. Gupta, and B. Friedrich, J. of Chem. Phys. 110, 3870 (1999). 
[48] C. B. Madsen and L. B. Madsen, Phys. Rev. A 76, 043419 (2007).

[49] J. Levesque, D. Zeidler, J. P. Marangos, P. B. Corkum, and D. M. Villeneuve, Phys. Rev. Lett. 98, 183903 (2007).

[50] R. E. Ziemer, W. H. Tranter, and D. R. Fannin, Signals and systems : continuous and discrete (Macmillan, New York, NY, USA, 1993), 3rd ed.

[51] R. W. Ramirez, The FFT, fundamentals and concepts (Prentice-Hall, Englewood Cliffs, NJ, USA, 1985).

[52] O. Smirnova, S. Patchkovskii, Y. Mairesse, N. Dudovich, D. Villeneuve, P. Corkum, and M. Y. Ivanov, Phys. Rev. Lett 102, 063601 (2009).

[53] W. Li, X. Zhou, R. Lock, S. Patchkovskii, A. Stolow, H. Kapteyn, and M. Murnane, Science 322, 1207 (2008).

[54] X. Zhou, R. Lock, N. Wagner, W. Li, H. C. Kapteyn, and M. M. Murnane, Phys. Rev. Lett. 102, 073902 (2009).

[55] V. I. Usachenko, P. E. Pyak, and S. I. Chu, Laser Physics 16, 1326 (2006). 